\title{
Instruments to measure fear of COVID-19: a diagnostic systematic review
}

Ashley Elizabeth Muller ${ }^{*}$ (D), Jan Peter William Himmels and Stijn Van de Velde

\begin{abstract}
Background: The COVID-19 pandemic has become a source of fear across the world. Measuring the level or significance of fear in different populations may help identify populations and areas in need of public health and education campaigns. We were interested in diagnostic tests developed to assess or diagnose COVID-19-related fear or phobia.
\end{abstract}

Methods: We performed a systematic review of studies that examined instruments diagnosing or assessing fear or phobia of COVID-19 (PROSPERO registration: CRD42020197100). We utilized the Norwegian Institute of Public Health's Live map of covid-19 evidence, a database of pre-screened and pre-categorized studies. The Live map of covid-19 evidence identified references published since 1 December 2019 in MEDLINE, Embase, and the Centers for Disease Control and Prevention. Following biweekly searches, two researchers independently categorized all studies according to topic (seven main topics, 52 subordinate topics), population (41 available groups), study design, and publication type. For this review, we assessed for eligibility all studies that had been categorized to the topic "Experiences and perceptions, consequences; social, political, economic aspects" as of 25 September 2020, in addition to hand-searching included studies' reference lists. We meta-analyzed correlation coefficients of fear scores to the most common reference tests (self-reports of anxiety, depression, and stress), and reported additional concurrent validity to other reference tests such as specific phobias. We assessed study quality using the QUADAS-2 for the minority of studies that presented diagnostic accuracy statistics.

Results: We found 18 studies that validated fear instruments. Fifteen validated the Fear of COVID-19 scale (FCV195). We found no studies that proposed a diagnosis of fear of COVID-19 or a threshold of significant/clinical versus non-significant/subclinical fear. Study quality was low, with the most common potential biases related to sampling strategy and un-blinded data analysis. The FSV-19S total score correlated strongly with severe phobia $(r=0.703$, $95 \% \mathrm{Cl} 0.634-0.761)$ in one study, and moderately with anxiety in a meta-analysis.

Conclusions: The accuracy of the FSV-19S needs to be measured further using fear-related reference instruments, and future studies need to provide cut-off scores and normative values. Further evaluation of the remaining three instruments is required.

Keywords: Fear, Psychometrics, Diagnostic accuracy, SARS-CoV-2

(c) The Author(s). 2021 Open Access This article is licensed under a Creative Commons Attribution 4.0 International License, which permits use, sharing, adaptation, distribution and reproduction in any medium or format, as long as you give appropriate credit to the original author(s) and the source, provide a link to the Creative Commons licence, and indicate if changes were made. The images or other third party material in this article are included in the article's Creative Commons licence, unless indicated otherwise in a credit line to the material. If material is not included in the article's Creative Commons licence and your intended use is not permitted by statutory regulation or exceeds the permitted use, you will need to obtain permission directly from the copyright holder. To view a copy of this licence, visit http://creativecommons.org/licenses/by/4.0/. The Creative Commons Public Domain Dedication waiver (http://creativecommons.org/publicdomain/zero/1.0/) applies to the data made available in this article, unless otherwise stated in a credit line to the data. 


\section{Background}

Fear is an emotional response to a threat as perceived by an individual, and considered a functional, adaptive, and transient response to stimuli, briefly resulting in physiological changes [1, 2]. Fear can become pathological when physiological changes are chronic instead of transient, when fear reactions are triggered in the absence of actual danger, and/or when an individual is unable to learn safety signals, that is, to inhibit fear reactions by understanding safety cues (see $[3,4]$ ).

Infectious diseases are a particularly salient source of fear because they are transmissible, imminent, and invisible [2], and the COVID-19 pandemic has become a source of fear across the world. Schimmenti and colleagues [5] have suggested four dialectical elements of COVID-19 fear: fear of and for one's body, as one is both a potential vector and victim; fear of and for others, also related to the tension of prescribed social distancing from important interpersonal relationship; fear of ignorance of the virus as well as knowledge, as information is required for protection but can also be overwhelming and anxiety-inducing; and fear of both personal action and inaction, related to the behavioral consequences of fear. More than four out of five respondents reported one of the respective fears in two large surveys that included 1421 Japanese workers [6] and 669 dental practitioners worldwide [7]. In a recent systematic review of the mental health impact of COVID-19, healthcare workers reported that fear tied to their professional responsibilities extended into their personal lives [8]. Fear of getting sick with COVID-19 at work because they were unable to protect themselves extended to fear of infecting family members at home. This review found that fear correlated with greater exposure risk, supporting the general understanding of fear to be an appropriate response to an external threat.

Several surveys have also demonstrated that those more directly impacted by the pandemic or those at risk of being personally impacted are more afraid, as seen in people currently laid off or furloughed [9], and in people at high risk due to comorbidities [6, 10]. Fear may have both positive consequences, such as greater adherence to infection prevention and control strategies, and negative consequences, such as avoiding health care services and settings. Harper et al. [11] recently reported a moderate positive correlation of fear to transmissionreducing behavior change such as hand-washing and social distancing $(r=0.31)$ in an international survey of 324 respondents. Karacin et al. [12] found that $14 \%$ of a Turkish center's oncology patients cited COVID-19 fear as the reason they delayed their chemotherapy (by an average of 47 days), despite no COVID-19 cases among patients or staff during the data collection period. Patients' and relatives' fear of infection was the overwhelming reason hypothesized by staff at 227 of China's 280 stroke hospitals - only half of which treated COVID-19 patients - to explain nationwide reductions in hospital admissions for thrombolysis/thrombectomy [13].

Individuals' fear can amass into critical social and public health problems. Taylor and colleague's early questionnaires reported that fear of COVID-19 was highly correlated with stigmatization of both healthcare workers and foreigners [14, 15]. These findings are an unfortunate reminder of Ebola outbreak research that pointed to fear-driven behavior with serious economic and social consequences, including stigmatization and discrimination of survivors [16-18]. Bali et al. [19] introduced the term "fearonomics" to describe these effects during Ebola. However, the threshold at which individuals' fear transforms into population-level problems is unclear: do a certain amount of people need to experience pathological or phobic fear, or a larger amount of people experiencing subclinical fear? Does the position of people in a society experiencing fear - e.g. a minority of policymakers versus a majority of voters - influence when fears become problematic on a population level?

Measuring the level or significance of fear in different populations may help identify populations and areas in need of public health and education campaigns. Several validated instruments are available to serve as gold standard reference tests to diagnosis phobic and nonphobic fears related to illness, such as the Illness Attitudes Scales [20], the Fear Survey Schedule III [21], and the Perceived Vulnerability to Disease [22]. These gold standard reference tests and others have been used in the development of fear scales of Ebola [23] and swine flu [24].

Many studies measuring fear of COVID-19 have used unvalidated instruments, although the Fear of COVID19 Scale [25] is increasingly used. It is imperative that fear of COVID-19 be measured appropriately. This systematic review examines scales used to assess or diagnosis fear of COVID-19.

\section{Method}

We conducted a systematic review according to our protocol registered in PROSPERO (CRD42020197100), and following Cochrane's guidelines for diagnostic accuracy tests.

\section{Inclusion criteria}

We included all studies that identified themselves as diagnostic accuracy or validation studies, and that measured the accuracy or validated the psychometric properties of any instrument measuring fear of COVID-19. Widening the selection of study designs beyond diagnostic accuracy studies was based on Umemneku Chikere 
and colleagues' recent systematic review of diagnostic accuracy tests conducted in the absence of a gold standard reference test, which found the use of validation studies to comprise one of four methodological alternatives [26]. We had no exclusion criteria related to population, intervention, comparator, outcome, or language; however, Chinese-language studies were not identified in our search strategy.

\section{Literature search and article selection}

We identified relevant studies by searching the Norwegian Institute of Public Health's (NIPH's) publicly available Live map of covid-19 evidence (https://www.fhi.no/ en/qk/systematic-reviews-hta/map/) on 25 September 2020. This map is one of several evidence maps and living evidence databases that attempt to enable faster identification of relevant publications with primary data. Not only has the speed with which covid-19 publications proceed through the peer-review and publication processes increased exponentially, even when compared to previous pandemics [27], the majority of publications on COVID-19 do not in fact present data [28]. Researchers and evidence synthesizers face significant challenges in identifying relevant publications, beginning simply by filtering out those lacking empirical data. Numerous initiatives have begun that use human effort, machine learning, or a combination, to identify relevant studies, categorize them according to intervention or population group, and in some cases extract and analyze data before a systematic review has been ordered, so that researchers will be positioned to rapidly produce high-quality evidence syntheses on a variety of topics as soon as policymakers request them. Some of the larger initiatives include the COVID-NMA project, focused on continuously updating analyses of randomized trials [29]; Epistemonikos' LOVE database, which categorizes studies according to "type of question", suitable for systematic reviews [30]; and the EPPI Centre's living map, containing 11 heterogenous and mutually exclusive categories such as vaccine development, genetics, and mental health impacts [31].

NIPH's Live map of covid-19 evidence is one of the most granular evidence maps, and all studies are categorized according to topic (seven main topics, 52 subordinate topics), population (41 available groups), study design, and publication type, by two researchers independently. The map's protocol describes the methodology in detail [32]. The search strategy of the Live map of covid-19 evidence has developed dynamically since March 2020, and is updated online [32]. The Live map of covid-19 evidence first searched for references on 12 March 2020, and identified references published since 1 December 2019 in MEDLINE, Embase, Centers for Disease Control and Prevention, and the Center for
Evidence-Based Medicine. EPPI Centre at University College London has conducted the majority of searches (MEDLINE and Embase) and screening from May 2020 and onwards [31].

For this systematic review, we identified references categorized to the topic "Experiences and perceptions, consequences; social, political, economic aspects". One researcher screened all identified references specifically for the inclusion criteria for this systematic review, and read in full-text those containing the words fear, phobi*, diagnos*, accuracy, psychometric*, validation, or scale, in title/abstract. We also hand-searched included studies' reference lists. On the search day, the map's website stated that all relevant studies identified in database searches on or before 4 May 2020 were categorized, along with systematic reviews, health technology assessments, and randomized and non-randomized controlled trials up until 24 August 2020.

\section{Data extraction and methodological quality assessment}

We developed a data extraction form to collect data on country, target group and participants (age, gender, COVID-19 characteristics and socioeconomic characteristics as reported by study author), instrument description, methods of accuracy/validity assessment (such as reference tests), and outcomes. One researcher extracted data, and a second researcher checked extraction for accuracy.

We planned on using the QUADAS-2 [33] for diagnostic accuracy studies to assess methodological quality. Only three studies [34-36] tested a fear instrument against a dichotomous reference test and could therefore be assessed as diagnostic accuracy studies; the remainder measured convergent validity to continuous scores of other patient-reported outcomes. We assessed only these three studies using the QUADAS-2.

\section{Data presentation and analyses}

While we intended to conduct a summary ROC analysis to assess sensitivity and specificity of fear instruments, only one of the included studies produced an area under the curve score. In lieu of a summary ROC analysis, we reported each study's validation assessments using classical test theory or Rasch analysis, namely measures of internal consistency reliability, factor structure, and goodness-of-fit tests (Additional file 1). We metaanalyzed correlation coefficients between total fear instrument scores and other reference tests' total scores. We reported the correlation of fear to other constructs reported by at least one study, and calculated 95\% confidence intervals. We conducted all analyses in STATA v.16 [37] using the metan command. 


\section{Results}

Results of the literature search

As of 25 September 2020, the Live map of covid-19 evidence project in cooperation with EPPI Centre had screened 56,977 studies for COVID-19 relevance, and categorized 9431 studies with empirical data, either primary, secondary, or modelled. Within the studies categorized to the topic Experiences, we identified 394 with a relevant keyword in title/abstract. One additional study was identified through hand-searching studies' references lists. After assessing these 395 studies for eligibility, 18 met our inclusion criteria [25, 35, 36, 38-52] (Fig. 1).

\section{Description of studies}

Eighteen studies were included, with a large geographic spread. Three studies took place in Turkey, two studies each in China, Peru, and Israel, and one each in Iran, Bangladesh, Italy, Saudi Arabia, Vietnam, Unites States, and Greece; one study sampled participants from both Belarus and Russia. All studies but one were online questionnaires that recruited via social media or used other convenience sampling methods. Most studies targeted the general population; four sampled among university students/graduates, one recruited participants working at public health departments, with no further description of sampling methods [45], and another

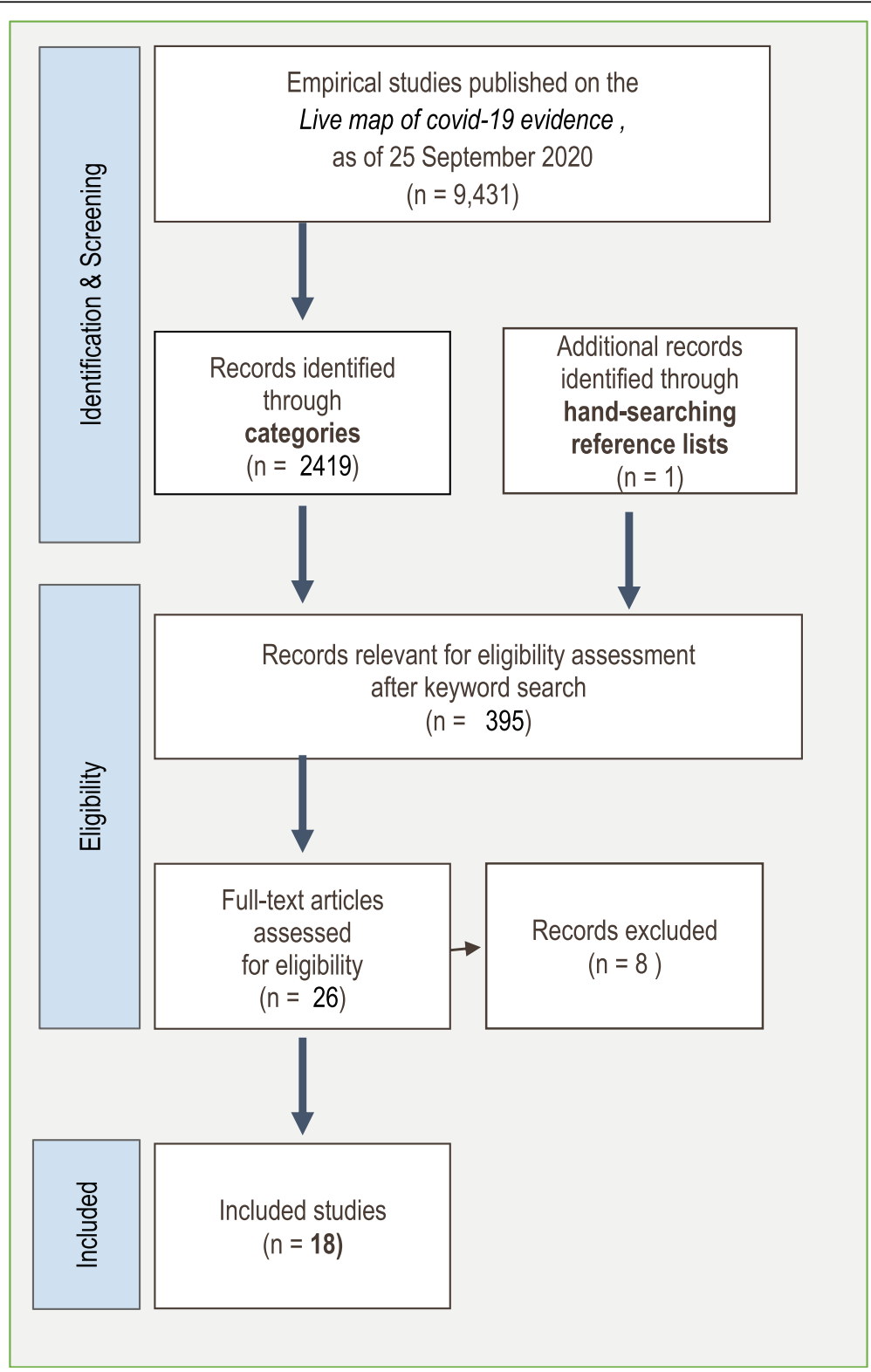

Fig. 1 Live evidence map flow diagram of study inclusion. As of 25 September 2020, the Live map of covid-19 evidence project in cooperation with EPPI Centre had categorized 9431 studies. Twenty-six were assessed in full-text for this review, and 18 included 
Table 1 Characteristics of the 18 included studies

\begin{tabular}{|c|c|c|c|c|}
\hline Study & Instrument & $\begin{array}{l}\text { Setting, target group, and participant } \\
\text { description }\end{array}$ & Language & Test accuracy or validity methods ${ }^{a}$ \\
\hline $\begin{array}{l}\text { Ahorsu et al. } \\
\text { [25] }\end{array}$ & $\begin{array}{l}\text { FCV-19S (Fear of COVID-19 } \\
\text { Scale) - development paper }\end{array}$ & $\begin{array}{l}\text { Iran } \\
N=717 \text { general population } \\
\text { Mean age: } 31.25 \text { years (SD 12.68), } \\
\text { Female: } 42.0 \% \\
\text { COVID-19 characteristics: NR } \\
\text { Socioeconomic: } 8.9 \text { years of education (SD } \\
\text { 4.1) }\end{array}$ & $\begin{array}{l}\text { Iranian, with } \\
\text { English } \\
\text { translation } \\
\text { provided }\end{array}$ & $\begin{array}{l}\text { Convergent validity (HADS Anxiety, HADS } \\
\text { Depression, PVDS). } \\
\text { Internal consistency reliability. }\end{array}$ \\
\hline $\begin{array}{l}\text { Alyami et al. } \\
{[38]}\end{array}$ & FCV-19S & $\begin{array}{l}\text { Saudi Arabia } \\
N=639 \text { general population } \\
\text { Mean age: } 34.75 \text { years (SD 11.80) } \\
\text { Female: } 42.1 \% \\
\text { COVID-19 characteristics: NR } \\
\text { Socioeconomic: } 70 \% \text { university } \\
\text { qualification; } 50.2 \% \text { employed, } 15.6 \% \\
\text { unemployed, } 27.4 \% \text { student }\end{array}$ & Arabic & $\begin{array}{l}\text { Convergent validity (HADS total score, } \\
\text { HADS Anxiety, HADS Depression). } \\
\text { Internal consistency reliability. }\end{array}$ \\
\hline $\begin{array}{l}\text { Arpaci et al. } \\
\text { [39] }\end{array}$ & $\begin{array}{l}\text { COVID-19 Phobia Scale } \\
\text { (C19P-S) - development } \\
\text { paper }\end{array}$ & $\begin{array}{l}\text { Turkey } \\
\text { Study 1: } N=1250 \text { general population } \\
\text { Mean age: } 37.53 \text { years (SD = 16.94) } \\
\text { Female: } 61.2 \% \\
\text { COVID-19 characteristics: } 0.4 \% \text { positive } \\
\text { Socioeconomic: } 1.8 \% \text { high, } 21.3 \% \text { middle- } \\
\text { high, } 57.5 \% \text { middle, } 14.6 \% \text { low. } \\
\text { Study 2: } N=2143 \text { general population } \\
\text { Mean age: } 39.66 \text { years (SD 16.87) } \\
\text { Female: } 60-3 \% \\
\text { COVID-19 characteristics: } 0.5 \% \text { positive } \\
\text { Socioeconomic: } 1.5 \% \text { high, } 20.2 \% \text { middle- } \\
\text { high, } 60.1 \% \text { middle, } 12.7 \% \text { middle-low, } \\
\text { 12.7\% low }\end{array}$ & $\begin{array}{l}\text { Turkish, with } \\
\text { English } \\
\text { translation } \\
\text { provided }\end{array}$ & $\begin{array}{l}\text { Reference test: COVID-19 infection vs not. } \\
\text { Internal consistency reliability. }\end{array}$ \\
\hline $\begin{array}{l}\text { Bitan et al. } \\
\text { [40] }\end{array}$ & FCV-19S & $\begin{array}{l}\text { Israel } \\
N=649 \\
\text { Mean age: NR } \\
\text { Female: } 84.8 \% \\
\text { COVID-19 characteristics: } 52.9 \% \\
\text { unemployed during lockdown, } 58.3 \% \text { main } \\
\text { career during COVID-19, } 18.0 \% \text { in risk } \\
\text { group for COVID-19 mortality and } 77.0 \% \\
\text { not in risk group, } 4.1 \% \text { direct contact with } \\
\text { COVID-19 patient, } 0.6 \% \text { COVID-19 death in } \\
\text { family } \\
\text { Socioeconomic: } 33.2 \% \text { above average, } \\
45.6 \% \text { below average, } 21.1 \% \text { average }\end{array}$ & Hebrew & $\begin{array}{l}\text { Convergent validity (DASS-21), internal } \\
\text { consistency reliability. }\end{array}$ \\
\hline $\begin{array}{l}\text { Chang et al. } \\
\text { [41] }\end{array}$ & FCV-19S & $\begin{array}{l}\text { Taiwan } \\
N=400 \text { adults receiving inpatient or } \\
\text { outpatient treatment for psychiatric } \\
\text { disorder } \\
\text { Mean age: } 46.91 \text { years (SD 10.92) } \\
\text { Female: } 44.5 \% \\
\text { COVID-19 status: NR } \\
\text { Socioeconomic: Mean education years } \\
\text { 11.31 (SD 2.98) }\end{array}$ & Chinese & Internal consistency reliability. \\
\hline $\begin{array}{l}\text { Feng et al. } \\
{[42]}\end{array}$ & $\begin{array}{l}\text { Scale of COVID-19 related } \\
\text { psychological distress in } \\
\text { healthy public (CORPD) - de- } \\
\text { velopment paper }\end{array}$ & $\begin{array}{l}\text { China } \\
N=652 \text { uninfected healthy people } \\
\text { Mean age: NR } \\
\text { Female: } 67.3 \% \\
\text { COVID-19 status: NR } \\
\text { Socioeconomic: } 82.8 \% \text { had college degree } \\
\text { or above, } 56.4 \% \text { had a monthly income of } \\
\text { more than } 4000 \text { yuan }\end{array}$ & Chinese & $\begin{array}{l}\text { Convergent validity (SCL-90), internal } \\
\text { consistency reliability: }\end{array}$ \\
\hline $\begin{array}{l}\text { Haktanir et al. } \\
{[43]}\end{array}$ & FCV-19S & $\begin{array}{l}\text { Turkey } \\
N=668 \text { general population } \\
\text { Mean age: NR } \\
\text { Female: } 72.0 \% \\
\text { COVID-19 status: NR }\end{array}$ & Turkish & $\begin{array}{l}\text { Convergent validity (BRS), internal } \\
\text { consistency reliability. }\end{array}$ \\
\hline
\end{tabular}


Table 1 Characteristics of the 18 included studies (Continued)

\begin{tabular}{|c|c|c|c|c|}
\hline Study & Instrument & $\begin{array}{l}\text { Setting, target group, and participant } \\
\text { description }\end{array}$ & Language & Test accuracy or validity methods ${ }^{a}$ \\
\hline & & $\begin{array}{l}\text { Socioeconomic: } 31.4 \% \text { high, } 61.4 \% \text { middle, } \\
7 \% \text { low }\end{array}$ & & \\
\hline $\begin{array}{l}\text { Huarcaya- } \\
\text { Victoria et al. } \\
{[44]}\end{array}$ & FCV-19S & $\begin{array}{l}\text { Peru } \\
N=832 \text { general population } \\
\text { Mean age: } 38.37 \text { years (SD 12.75) } \\
\text { Female: } 65.6 \% \\
\text { COVID-19 status: } 68.4 \% \text { without any } \\
\text { symptoms of COVID-19, } 21.0 \% \text { with one } \\
\text { symptom, } 6.5 \% \text { with two symptoms, } 3.5 \% \\
\text { with three symptoms, } 0.6 \% \text { with four or } \\
\text { more symptoms } \\
\text { Socioeconomic: } 76.4 \% \text { with university } \\
\text { education, } 66.9 \% \text { with formal employment }\end{array}$ & Spanish & $\begin{array}{l}\text { Convergent validity (GAD-7, PHQ-9, IES-R), } \\
\text { internal consistency reliability: }\end{array}$ \\
\hline $\begin{array}{l}\text { Mejia et al. } \\
{[45]}\end{array}$ & $\begin{array}{l}\text { Fear Perception and } \\
\text { Magnitude of the Issue } \\
\text { (MED-COVID-19) - } \\
\text { development paper }\end{array}$ & $\begin{array}{l}\text { Peru } \\
N=\text { about } 400 \text { public employees } \\
\text { Mean age: NR } \\
\text { Female: NR } \\
\text { COVID-19 status: NR } \\
\text { Socioeconomic: NR }\end{array}$ & $\begin{array}{l}\text { Spanish, } \\
\text { Portuguese }\end{array}$ & Internal consistency reliability \\
\hline $\begin{array}{l}\text { Nguyen et al. } \\
\text { [35] }\end{array}$ & FCV-19S & $\begin{array}{l}\text { Vietnam } \\
N=5423 \text { university students } \\
\text { Mean age: } 22.0 \text { years (SD 2.0) } \\
\text { Female: } 52.0 \% \\
\text { COVID-19 status: } 18.9 \% \text { with suspected } \\
\text { symptoms, } 81.0 \% \text { without suspected } \\
\text { symptoms } \\
\text { Socioeconomic: } 53.9 \% \text { with very or fairly } \\
\text { easy ability to pay for medication, } 46.0 \% \\
\text { very or fairly difficult ablity to pay }\end{array}$ & Vietnamese & $\begin{array}{l}\text { Reference test: AUC to distinguish GAD } \geq 8 \text {. } \\
\text { Internal consistency reliability: }\end{array}$ \\
\hline $\begin{array}{l}\text { Pang et al. } \\
\text { [46] }\end{array}$ & FCV-19S & $\begin{array}{l}\text { Malaysia } \\
N=228 \text { university students } \\
\text { Mean age: NR } \\
\text { Female: } 71.1 \% \\
\text { COVID-19 status: NR } \\
\text { Socioeconomic: } 1.8 \% \text { doctoral degree, } \\
5.7 \% \text { master degree, } 56.1 \% \text { bachelor } \\
\text { degree, } 26.8 \% \text { diploma, } 9.6 \% \text { high school }\end{array}$ & Malay & $\begin{array}{l}\text { Convergent validity (DASS-21), internal } \\
\text { consistency reliability. }\end{array}$ \\
\hline $\begin{array}{l}\text { Perz et al. } \\
{[47]}\end{array}$ & FCV-19S & $\begin{array}{l}\text { United States } \\
N=237 \text { university students } \\
\text { Mean age: } 30.3 \text { years (SD 10.2) } \\
\text { Female: } 73.0 \% \\
\text { COVID-19 status: } 29 \% \text { know someone with } \\
\text { COVID-19 symptoms } \\
\text { Socioeconomic: } 73 \% \text { negative financial } \\
\text { impact by COVID-19 or response }\end{array}$ & English & $\begin{array}{l}\text { Convergent validity (GAD-7), internal } \\
\text { consistency validity }\end{array}$ \\
\hline $\begin{array}{l}\text { Reznik et al. } \\
{[48]}\end{array}$ & FCV-19S & $\begin{array}{l}\text { Belarus and Russia } \\
N=850 \text { university students/ graduates } \\
\text { Mean age: } 34.8 \text { (SD 13.0) } \\
\text { Female: } 73.2 \% \\
\text { COVID-19 status: NR } \\
\text { Socioeconomic: } 65.4 \% \text { university graduate, } \\
28.4 \% \text { university student, } 6.2 \% \text { primary or } \\
\text { secondary school education }\end{array}$ & Russian & internal consistency reliability \\
\hline $\begin{array}{l}\text { Sakib et al. } \\
{[49]}\end{array}$ & FCV-19S & $\begin{array}{l}\text { Bangladesh } \\
N=8550 \text { general population } \\
\text { Mean age: } 26.5 \text { years (SD 9.1) } \\
\text { Female: } 44.0 \% \\
\text { COVID-19 status: NR } \\
\text { Socioeconomic: } 82.0 \% \text { educated at tertiary } \\
\text { level, } 59.6 \% \text { student, } 3.7 \% \text { unemployed }\end{array}$ & Bangal & $\begin{array}{l}\text { Convergent validity (PHQ-9), internal } \\
\text { consistency reliability }\end{array}$ \\
\hline $\begin{array}{l}\text { Satici et al. } \\
{[50]}\end{array}$ & FCV-19S & $\begin{array}{l}\text { Turkey } \\
N=1304 \text { general population }\end{array}$ & Turkish & $\begin{array}{l}\text { Convergent validity (DASS-21, SWLS), } \\
\text { internal consistency reliability }\end{array}$ \\
\hline
\end{tabular}


Table 1 Characteristics of the 18 included studies (Continued)

\begin{tabular}{|c|c|c|c|c|}
\hline Study & Instrument & $\begin{array}{l}\text { Setting, target group, and participant } \\
\text { description }\end{array}$ & Language & Test accuracy or validity methods ${ }^{a}$ \\
\hline & & $\begin{array}{l}\text { Female: } 70.3 \% \\
\text { COVID-19 status: } 1.7 \% \text { symptoms, } 12.9 \% \\
\text { partial symptoms, } 85.4 \% \text { no symptoms } \\
\text { Socioeconomic: } 10.0 \% \text { graduate degree, } \\
79.8 \% \text { bachelor degree, } 3.8 \% \text { associate } \\
\text { degree, } 5.0 \% \text { high school, } 1.4 \text { less than } \\
\text { high school }\end{array}$ & & \\
\hline $\begin{array}{l}\text { Soraci et al. } \\
\text { [51] }\end{array}$ & FCV-19S & $\begin{array}{l}\text { Italy } \\
N=249 \text { general population } \\
\text { Mean age: } 34.50 \text { years (SD 12.21) } \\
\text { Female: } 92 \% \\
\text { COVID-19 status: NR } \\
\text { Socioeconomic: } 58.7 \% \text { university-level de- } \\
\text { gree, } 39 \% \text { high school degree, } 2.4 \% \text { lower- } \\
\text { level educational degree }\end{array}$ & Italian & $\begin{array}{l}\text { Convergent validity (HADS, SMSP-A), in- } \\
\text { ternal consistency reliability }\end{array}$ \\
\hline $\begin{array}{l}\text { Tsipropoulou } \\
\text { et al. [52] }\end{array}$ & FCV-19S & $\begin{array}{l}\text { Greece } \\
N=2970 \text { general population } \\
\text { Mean age: NR } \\
\text { Female: } 72.5 \% \\
\text { COVID-19 status: NR } \\
\text { Socioeconomic: } 45.4 \% \text { university degree, } \\
29.8 \% \text { high school degree, } 1.5 \% \text { less than } \\
\text { high school. } 8.9 \% \text { health care provider. }\end{array}$ & Greek & $\begin{array}{l}\text { Convergent validity (PHQ-9, GAD-7), in- } \\
\text { ternal consistency reliability }\end{array}$ \\
\hline $\begin{array}{l}\text { Zolotov et al. } \\
\text { [36] }\end{array}$ & FCV-19S & $\begin{array}{l}\text { Israel } \\
N=370 \text { university students } \\
\text { Mean age: } 25.2 \text { (SD 3.1) } \\
\text { Female: } 78.1 \% \\
\text { COVID-19 status: NR } \\
\text { Socioeconomic: NR }\end{array}$ & Hebrew & $\begin{array}{l}\text { Reference test: convergent validity to } \\
\text { single items regarding COVID-19-related } \\
\text { depression, anxiety, nervousness, loneli- } \\
\text { ness, and exhaustion. Internal consistency } \\
\text { reliability: }\end{array}$ \\
\hline
\end{tabular}

NR Not reported. Other instruments: HADS Hospital Anxiety and Depression Scale, SMSP-A Severity Measure for Specific Phobia- Adult, PHQ-9:, DASS.21:, GAD-7 Generalized Anxiety Disorder scale, BRS Brief Resilience Scale, SCL-90 Symptom Checklist-90, PVDS Perceived Vulnerability to Disease Scale

${ }^{a}$ Convergent validity is displayed in forest plots in Figs. 2, 3, 4 and 5. Other validity assessments are in Additional file 1

recruited patients receiving psychiatric treatment [41]. No studies were conducted specifically among healthcare personnel. Sample sizes ranged from 228 to 8550 participants (Table 1).

\section{Instruments}

The eighteen included studies validated four instruments. No study validated more than one instrument.

\section{Fear of COVID-19 Scale (FCV-19S)}

Fifteen studies validated the Fear of COVID-19 Scale (FCV-19S), including the original development study. This seven-item instrument was developed by Ahorsu et al. [25]. The authors first identified thirty general instruments on fear through a literature review, pooled and de-duplicated relevant items, solicited expert input to further reduce the amount of items, piloted the resulting ten-item instrument among 46 non-clinical participants for feasibility, administered it among a larger sample $(N=717)$, and finally, removed three items following classical test theory analysis. Respondents indicate their agreement with each question, as for example "I am afraid of losing my life because of coronavirus", on a 1-5 Likert-type scale from "strongly disagree" to "strongly agree". All answers are summed to produce a total score, from 7 to 35 , with higher scores indicating more fear.

While the original authors and eleven subsequent studies concluded the FCV-19S was unidimensional, Bitan et al. [40] found a two-factor fit of emotional fear and symptomatic expressions of fear. Huarcaya-Victorial et al. [44] found a bifactor model, in which all seven items load onto one general factor and onto one of two subordinate factors of emotional fear and symptomatic expressions, as in Bitan et al. Zolotov et al. reported weak fit indices for a one-factor and a two-factor model, but did not report the items comprising the two factors. With the exception of Zolotov et al., all studies utilizing classical test theory or Rasch analysis reported a stable factor structure, and adequate fit indices (see Additional file 1).

Most studies that used reference tests used validated and patient-reported screening measures of symptom severity. No studies used fear diagnoses as reference tests. Nguyen et al. [35] reported the area under the curve and 95\% confidence intervals to predict clinically significant anxiety on the Generalized Anxiety Disorder Screener (scores $\geq 8)$. The area under the curve was $0.63(0.60-$ 
0.66); meaning FCV-19S scores correctly distinguished participants with clinically significant anxiety from clinically non-significant anxiety in $63 \%$ of cases. Zolotov et al.'s [36] reference tests were self-reports of how much more depressed, anxious, nervous, exhausted, or lonely participants felt in the past month during the COVID-19 pandemic; results are presented under the sub-headings of anxiety and depression.

Thirteen studies tested convergent/concurrent validity to reference tests with continuous scores as a validation method, with all reporting significant bivariate correlations between the FCV-19 total score (and each subscore, in the case of Bitan et al. [40]; not reported) and the various psychological constructs tested (Fig. 2).

Across nine studies, greater anxiety correlated with greater fear on the FSV-19S ( $r=0.55$, CI 0.46-0.64), as displayed in Fig. 2. Zolotov et al. [36] measured anxiety dichotomously, and found that the total FSV-10S score distinguished between self-reports of feeling more anxious as a result of COVID-19 in the past month, versus not.

Figure 3 shows that greater depression also correlated with greater fear $(r=0.4$, CI 0.34-0.46). The total score distinguished between participants who reported feeling more depressed as a result of the pandemic in the past month and those who did not report feeling more depressed, reported by Zolotov et al. [36].

Stress was measured in three studies (Fig. 4), and more stress correlated with more fear $(r=0.39$, CI 0.28-0.50).
The Hospital Anxiety and Depression Scale total score, a measure of general distress, was correlated with the FSV-19S ( $r=0.60$, CI 0.51-0.69) (Fig. 5).

Each meta-analysis contained a very large amount of unexplained heterogeneity, a strong suggestion that the studies' populations, contexts, or methods were not similar enough to combine. It is therefore useful for readers to visually examine individual studies' results in each forest plot.

Concurrent validity was suggested according to significant correlations between the FSV-19S total score and perceived infectability from the Perceived Vulnerability to Disease Scale $(r=0.483$, CI $0.425-0.537)$, and germ aversion from the same scale $(r=0.459$, CI $0.400-0.512)$ in Ahorsu et al.'s development study [25]. Severe phobia also correlated positively with FSV-19S total score $(r=$ 0.703, CI 0.634-0.761) [51].

More resilience was associated with less fear $(r=0.32$, $\mathrm{CI}-.386$ to -.250 ) in Haktanir et al. [43], and greater life satisfaction was associated with less fear $(r=-0.200, \mathrm{CI}$ -.252 to -.147 ) in Satici et al. [50].

\section{Fear perception and magnitude of the issue (MED-COVID- 19) scale}

The MED-COVID-19 was developed by a group of experts, piloted, and administered to approximately 400 public employees in Peru [45]. Only one validation study was identified for this instrument, which measured both the extent of fear and fear sources, such as media,

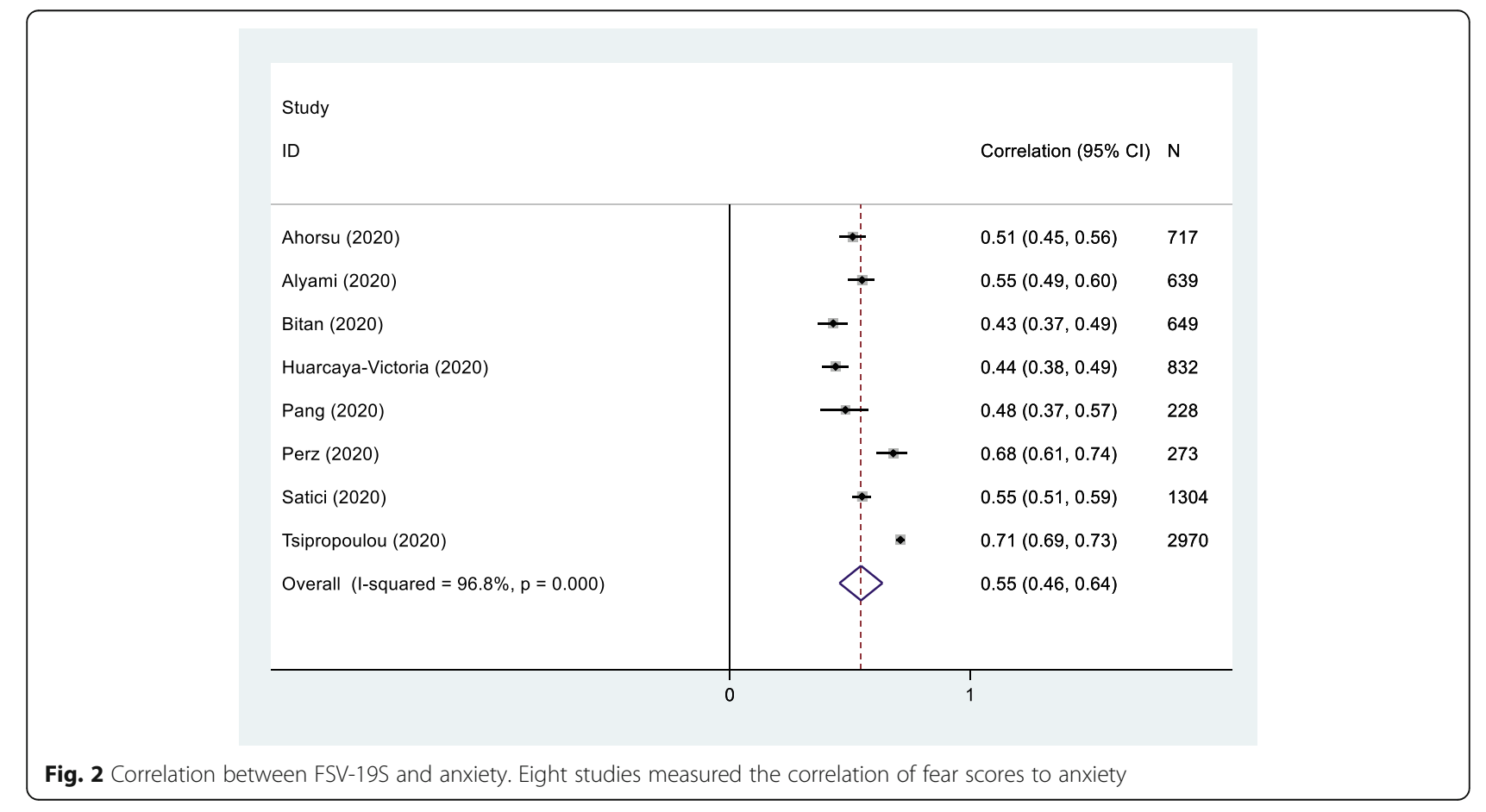




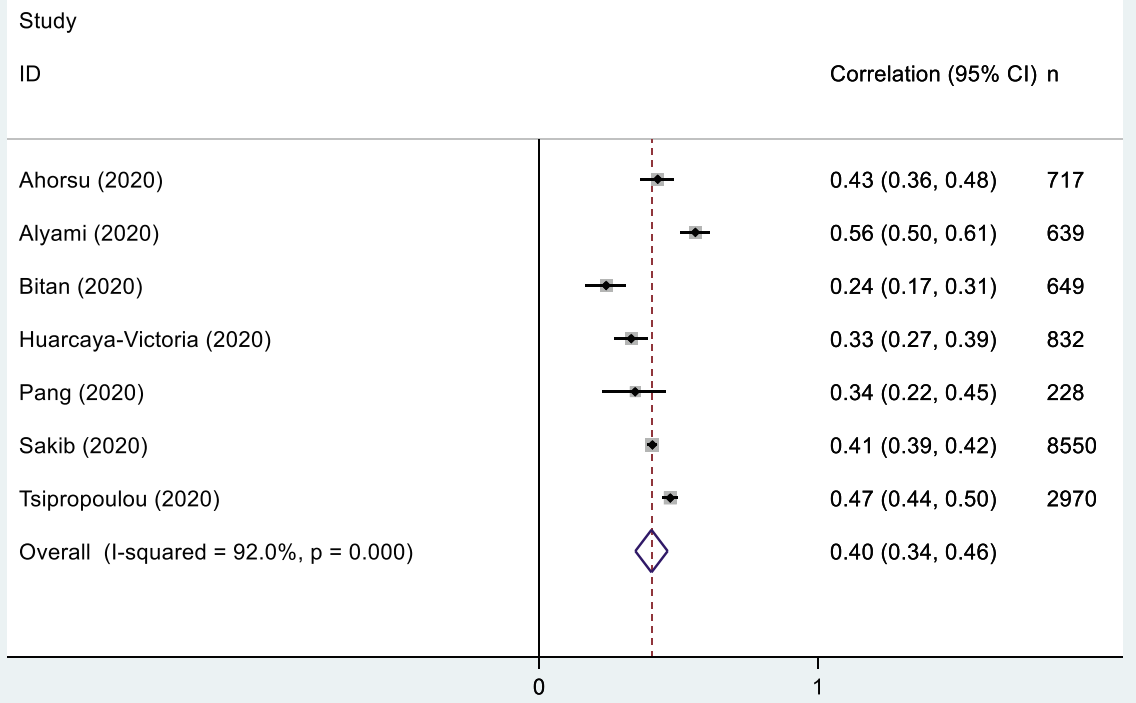

Fig. 3 Correlation between FSV-19S and depression. Seven studies measured the correlation of fear scores to depression

healthcare providers, and family/friends. Three factors were uncovered within twelve items through Rasch analysis. The first factor contained items relating to the perception that media sources were exaggerating COVID19 , the second factor related to magnitude of fear from media, and the third factor to both perception and magnitude of fear arising from healthcare providers, family, and friends. The validation study did not assess the
MED-COVID-19 against a reference test, assess concurrent validity, or report subscale scores or total scores.

\section{Scale of COVID-19 Related Psychological Distress in the} healthy public (CORPD)

The Scale of COVID-19 [42] related psychological distress in the healthy public (CORPD), consists of two factors: fear/anxiety and suspicion. Both dimensions had

Study

ID

Correlation $(95 \% \mathrm{Cl}) \quad \mathrm{n}$

\begin{tabular}{llll}
\hline Bitan (2020) & $0.31(0.24,0.38)$ & 649 \\
Satici (2020) & $0.47(0.43,0.51)$ & 1304 \\
Pang (2020) & $0.39(0.27,0.49)$ & 228 \\
Overall (I-squared $=86.9 \%, p=0.000)$ & $0.39(0.28,0.50)$ \\
& 0 & & \\
\hline
\end{tabular}

Fig. 4 Correlation between FSV-19S and stress. Three studies measured the correlation of fear scores to stress 
ID

Correlation $(95 \% \mathrm{Cl}) \mathrm{n}$

Soraci $(2020)$

Soraci (2020)

Huarcaya-Victoria (2020)

Overall (I-squared $=75.6 \%, p=0.043$ )

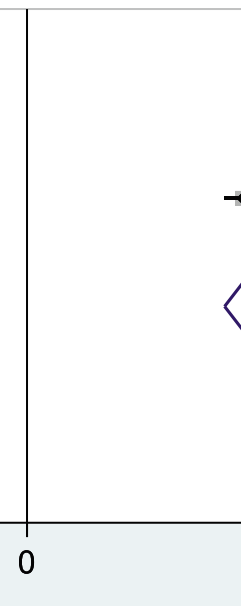

$\vdots$

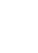


Table 2 Methodological quality assessment with QUADAS-2

\begin{tabular}{|c|c|c|c|c|c|c|c|c|c|c|c|c|c|c|c|c|c|c|c|}
\hline & \multicolumn{5}{|c|}{ Patient selection } & \multicolumn{4}{|c|}{ Index test } & \multicolumn{4}{|c|}{ Reference standard } & \multicolumn{5}{|c|}{ Flow and timing } & \multirow{2}{*}{\begin{tabular}{|l} 
Overall \\
quality \\
\end{tabular}} \\
\hline & 1 & 2 & 3 & 4 & 5 & 6 & 7 & 8 & 9 & 10 & 11 & 12 & 13 & 14 & 15 & 16 & 17 & 18 & \\
\hline \multicolumn{20}{|c|}{ Studies validating Fear of COVID-19 Scale (FCV-19S) } \\
\hline Ahorsu (2020) & & & & & & & & & & & & & & & & & & & $\mathrm{N} / \mathrm{A}$ \\
\hline Alyami (2020) & & & & & & & & & & & & & & & & & & & $\mathrm{N} / \mathrm{A}$ \\
\hline Bitan (2020) & & & & & & & & & & & & & & & & & & & $\mathrm{N} / \mathrm{A}$ \\
\hline Chang (2020) & & & & & & & & & & & & & & & & & & & $\mathrm{N} / \mathrm{A}$ \\
\hline Haktanir (2020) & & & & & & & & & & & & & & & & & & & $\mathrm{N} / \mathrm{A}$ \\
\hline $\begin{array}{l}\text { Huarcaya- } \\
\text { Victoria (2020) }\end{array}$ & & & & & & & & & & & & & & & & & & & $\mathrm{N} / \mathrm{A}$ \\
\hline Nguyen (2020) & - & + & + & - & + & - & $?$ & + & + & + & - & $\overline{-}$ & + & + & + & + & $?$ & $?$ & - \\
\hline Pang (2020) & & & & & & & & & & & & & & & & & & & N/A \\
\hline Perz (2020) & & & & & & & & & & & & & & & & & & & N/A \\
\hline Reznik (2020) & & & & & & & & & & & & & & & & & & & $\mathrm{N} / \mathrm{A}$ \\
\hline Sakib (2020) & & & & & & & & & & & & & & & & & & & $\mathrm{N} / \mathrm{A}$ \\
\hline Satici (2020) & & & & & & & & & & & & & & & & & & & $\mathrm{N} / \mathrm{A}$ \\
\hline Soraci (2020) & & & & & & & & & & & & & & & & & & & $\mathrm{N} / \mathrm{A}$ \\
\hline $\begin{array}{l}\text { Tsipropoulou } \\
(2020)\end{array}$ & & & & & & & & & & & & & & & & & & & $\mathrm{N} / \mathrm{A}$ \\
\hline Zolotov (2020) & $?$ & + & $?$ & - & + & - & $?$ & + & + & + & - & + & + & + & + & + & $?$ & $?$ & - \\
\hline \multicolumn{20}{|c|}{ Study validating Scale of COVID-19 related psychological distress in healthy public (CORPD) } \\
\hline Feng (2020) & & & & & & & & & & & & & & & & & & & $\mathrm{N} / \mathrm{A}$ \\
\hline \multicolumn{20}{|c|}{ Study validating MED-COVID-19 Scale } \\
\hline Mejia (2020) & & & & & & & & & & & & & & & & & & & $\mathrm{N} / \mathrm{A}$ \\
\hline \multicolumn{20}{|c|}{ Study validating Coronavirus 19 Phobia Scale (C19P-S). } \\
\hline Arpaci (2020) & $?$ & + & $?$ & - & ? & - & $?$ & + & + & $?$ & $?$ & $?$ & + & $?$ & $?$ & ? & ? & ? & - \\
\hline \multicolumn{20}{|c|}{$\begin{array}{l}\text { QUADAS-2 items: } \\
\text { 1. Was a consecutive or random sample of patients enrolled? } \\
\text { 2. Was a case-control design avoided? } \\
\text { 3. Did the study avoid inappropriate exclusions? } \\
\text { 4. Could the selection of patients have introduced bias? } \\
\text { 5. Is there concern that the included patients do not match the review question? } \\
\text { 6. Were the index test results interpreted without knowledge of the results of the reference standard? } \\
\text { 7. If a threshold was used, was it pre-specified? } \\
\text { 8. Could the conduct or interpretation of the index test have introduced bias? } \\
\text { 9. Is there concern that the index test, its conduct, or interpretation differ from the review question? } \\
\text { 10. Is the reference standard likely to correctly classify the target condition? } \\
\text { 11. Were the reference standard results interpreted without knowledge of the results of the index test? } \\
\text { 12. Could the reference standard, its conduct, or its interpretation have introduced bias? } \\
\text { 13. Is there concern that the target condition as defined by the reference standard does not match the review question? } \\
\text { 14. Was there an appropriate interval between index test(s) and reference standard? } \\
\text { 15. Did all patients receive a reference standard? } \\
\text { 16. Did patients receive the same reference standard? } \\
\text { 17. Were all patients included in the analysis? } \\
\text { 18. Could the patient flow have introduced bias? }\end{array}$} \\
\hline
\end{tabular}

psychological distress in healthy public (CORPD), and one study from Turkey validated the Coronavirus 19 Phobia Scale (C19P-S).
All but seven studies used anxiety as a reference test. Only two $[25,51]$ used disease fear or phobia-specific reference tests, and it is noteworthy that the highest 
correlation coefficient was between a self-reported measure of specific phobia $(r=0.703 \mathrm{CI} 0.634-0.761)$ and fear (FSV-19S total score). The only study that reported a standard diagnostic accuracy metric used FSV-19S to discriminate among clinically significant vs nonsignificant self-reported anxiety scores, which was possible in only $63 \%$ of cases: a poor level of accuracy [53]. Accuracy may have been higher had a fear, not anxiety, reference test been used. It is likely that sampling procedures and data collection modes - mainly convenience samples recruited through online social media - necessitated administration of simpler and non-diagnostic reference tests. The high amounts of unexplained heterogeneity in meta-analyses may be partially accounted for by the variety of reference tests. Heterogeneity may also be explained by an unstable factor structure. While twelve studies reported the FSV-19 to be unidimensional, one study found a two-factor fit and another a bi-factor fit; further research needs to explore dimensionality. As Schimmenti and colleagues [54] and Mertens and colleagues [55] have noted, fear may be more than pathological. Socioeconomic and interpersonal aspects of fear may be distinct factors or mediators of physiological manifestations of fear, and it is crucial that fear instruments are able to stably measure these dimensions.

The authors of included studies were able to quickly administer and collect data by choosing online convenience sampling, but therefore introduced potential sampling bias that reduced methodological quality in the three studies assessed with the QUADAS-2. The evidence so far supports the continued exploration of the FSV-19S based on a high correlation to severe phobia, and a moderate correlation to anxiety. To increase trustworthiness in these results, future studies should confirm this among non-selected samples, and should utilize fear-specific reference tests. The available evidence on the additional three instruments is not robust enough for recommending their use. The C19P-S was recently validated among an American sample in a forthcoming article (personal communication with I. Arpaci); depending on the findings, it may be a more appropriate instrument that uses the DSM-5-specific phobia criteria to measure COVID-19 fear.

While fear is understood to be adaptive and transient, and an appropriate response to a threat, Ebola research has emphasized the negative effects of disease-related fear on the community and international level [56]. Individual fear of infection and fear resulting from witnessing disease progression and death can turn into "a cyclical pattern of fear" on the community level (p.211), in which normal community interactions are disrupted because individuals loose trust in health services and reproduce stigma against infected individuals and survivors. In the current pandemic, discrimination and hate crimes against Asians have already been reported in North America [57] and Europe [58, 59], a worrying reminder of the stigma and discrimination that Africans reported globally during the 2013/2014 Ebola outbreak [56]. Fear of COVID-19 is increasingly being reported in systematic reviews related to mental health as a distinct outcome [60] or an identified risk factor for mental health problems [61]. With methodologically strong instruments, fear of COVID-19 can be measured and populations with more fear identified to receive public education and public health campaigns.

\section{Strengths and weaknesses}

This review is the first that we know of to systematically search for and assess diagnostic accuracy or other validation studies of fear of COVID-19 instruments. Our quality assessment of studies should help other researchers in the evidence synthesis process, if they choose to use methodological quality in their inclusion criteria. An additional methodological strength is our utilization of the publicly available Live map of covid-19 evidence, one of the first reviews to do so (others include Muller at al. [8], as well as a diagnostic accuracy review that used the map as one of multiple sources [62]). By using this map, we quickly identified 394 studies that had already been categorized to our topic of interest, without having to search in academic databases and screen again. Although this timely approach has increased the availability of overarching research, it includes the risk of missing relevant studies missing if they were not grouped to the relevant categories. We did not use any of the methodological shortcuts recently reported in a survey of rapid diagnostic review study authors [63].

\section{Conclusion}

At least four instruments have been developed to measure fear of COVID-19, ten months after the pandemic began. Most studies are assessing the validity of instruments against other patient-reported mental health problems, rather than measuring diagnostic accuracy against fear-related reference tests. The Fear of COVID19 scale (FCV-19S) instrument has already been translated to 13 languages, and our included studies reported convergent validity to phobia, disease-related fear, and anxiety. Future studies should assess diagnostic accuracy against validated fear-specific reference tests.

\section{Abbreviations}

COVID-19: Coronavirus disease 2019; CORPD: Scale of COVID-19 Related Psychological Distress in the Healthy Public; DSM: Diagnostic and Statistical Manual of Mental Disorders; FCV-19S: Fear of COVID-19 scale; MED-COVID19: Fear Perception and Magnitude of the Issue scale 


\section{Supplementary Information}

The online version contains supplementary material available at https://doi. org/10.1186/s12874-021-01262-5.

Additional file 1: Table 1. Validity outcomes.

\section{Acknowledgements}

The authors thank the international team supporting the Live map of covid19 evidence, particularly the project leader, Gunn Vist, and the numerous volunteer coders.

\section{Authors' contributions}

AEM conceived of the paper, AEM and JPWH extracted data and assessed methodological quality, SV and AEM drafted the analysis plan, and AEM conducted the analysis. All authors contributed to the first draft, contributed substantially to the final version, and read and approved the final version.

\section{Funding}

No funding was received.

\section{Availability of data and materials}

Data may be available by the authors upon request.

\section{Declarations}

Ethics approval and consent to participate

No ethical approval was required for this systematic review.

\section{Consent for publication}

Not applicable.

\section{Competing interests}

The authors declare no competing interests.

Received: 18 January 2021 Accepted: 31 March 2021

Published online: 23 April 2021

\section{References}

1. Adolphs R. The biology of fear. Curr Biol. 2013;23(2):R79-93. https://doi.org/1 0.1016/j.cub.2012.11.055

2. Pappas G, Kiriaze IJ, Giannakis P, Falagas ME. Psychosocial consequences of infectious diseases. Clin Microbiol Infect. 2009;15(8):743-7. https://doi.org/1 0.1111/j.1469-0691.2009.02947.x

3. Singewald N, Schmuckermair C, Whittle N, Holmes A, Ressler KJ. Pharmacology of cognitive enhancers for exposure-based therapy of fear, anxiety and trauma-related disorders. Pharmacol Ther. 2015;149:150-90. https://doi.org/10.1016/j.pharmthera.2014.12.004.

4. Jovanovic T, Kazama A, Bachevalier J, Davis M. Impaired safety signal learning may be a biomarker of PTSD. Neuropharmacology. 2012;62(2):695704. https://doi.org/10.1016/..neuropharm.2011.02.023.

5. Schimmenti A, Billieux J, Starcevic V. The four horsemen of fear: an integrated model of understanding fear experiences during the covid-19 pandemic. Clin Neuropsychiatry. 2020;17(2):4.

6. Sasak N, Kuroda R, Tsuno K, Kawakami N. Fear, worry and workplace harassment related to the COVID-19 epidemic among employees in Japan: prevalence and impact on mental and physical health; 2020.

7. Ahmed MA, Jouhar R, Ahmed N, Adnan S, Aftab M, Zafar MS, et al. Fear and Practice Modifications among Dentists to Combat Novel Coronavirus Disease (COVID-19) Outbreak. Int J Environ Res Public Health. 2020;17(8):111

8. Muller AE, Hafstad EV, Himmels JPW, Smedslund G, Flottorp S, Stensland S $\varnothing$, et al. The mental health impact of the covid-19 pandemic on healthcare workers, and interventions to help them: A rapid systematic review. Psychiatry Res. 2020:293:113441.

9. Fitzpatrick KM, Harris C, Drawve G. Fear of COVID-19 and the mental health consequences in America. Psychol Trauma. 2020;12(S1):S17-21. https://doi. org/10.1037/tra0000924.

10. Casanova M, Pagani Bagliacca E, Silva M, Patriarca C, Veneroni L, Clerici CA et al. How young patients with cancer perceive the COVID-19 (coronavirus) epidemic in Milan, Italy: is there room for other fears? Pediatr Blood Cancer. 2020;67(7):e28318

11. Harper CA, Satchell LP, Fido D, Latzman RD. Functional fear predicts public health compliance in the COVID-19 pandemic. Int J Ment Heal Addict. 2020: $1-14$.

12. Karacin C, Bilgetekin I, Basal FB, Oksuzoglu OB. How does COVID-19 fear and anxiety affect chemotherapy adherence in patients with cancer. Future Oncol. 2020;16(29):2283-93. https://doi.org/10.2217/fon-2020-0592.

13. Zhao J, Li H, Kung D, Fisher M, Shen Y, Liu R. Impact of the COVID-19 epidemic on stroke care and potential solutions. Stroke. 2020;51(7):19962001. https://doi.org/10.1161/STROKEAHA.120.030225.

14. Taylor S, Landry CA, Rachor GS, Paluszek MM, Asmundson GJG. Fear and avoidance of healthcare workers: an important, under-recognized form of stigmatization during the COVID-19 pandemic. J Anxiety Disord. 2020;75: 102289. https://doi.org/10.1016/j.janxdis.2020.102289.

15. Taylor S, Landry CA, Paluszek MM, Rachor GS, Asmundson GJG. Worry, avoidance, and coping during the COVID-19 pandemic: a comprehensive network analysis. J Anxiety Disord. 2020;76:102327. https://doi.org/10.1016/j. janxdis.2020.102327.

16. Jalloh MF, Li W, Bunnell RE, Ethier KA, O'Leary A, Hageman KM, et al. Impact of Ebola experiences and risk perceptions on mental health in Sierra Leone, July 2015. BMJ Glob Health. 2018;3(2):e000471. https://doi.org/10.1136/ bmigh-2017-000471.

17. Karafillakis E, Jalloh MF, Nuriddin A, Larson HJ, Whitworth J, Lees S, et al. 'Once there is life, there is hope' Ebola survivors' experiences, behaviours and attitudes in Sierra Leone, 2015. BMJ Glob Health. 2016;1(3):e000108.

18. O'Leary A, Jalloh MF, Neria Y. Fear and culture: contextualising mental health impact of the 2014-2016 Ebola epidemic in West Africa. BMJ Glob Health. 2018;3(3):e000924.

19. Bali S, Stewart KA, Pate MA. Long shadow of fear in an epidemic: fearonomic effects of Ebola on the private sector in Nigeria. BMJ Glob Health. 2016;1(3):e000111. https://doi.org/10.1136/bmjgh-2016-000111.

20. Sirri L, Grandi S, Fava GA. The illness attitude scales. Psychother Psychosom. 2008:77(6):337-50. https://doi.org/10.1159/000151387.

21. Beck JG, Carmin CN, Henninger NJ. The utility of the fear survey schedule-III. Anxiety Disord. 1998;12(3):177-82. https://doi.org/10.1016/S0887-61 85(98)00007-3.

22. Duncan LA, Schaller M, Park JH. Perceived vulnerability to disease: development and validation of a 15-item self-report instrument. Personal Individ Differ. 2009;47(6):541-6. https://doi.org/10.1016/j.paid.2009.05.001.

23. Blakey SM, Reuman L, Jacoby RJ, Abramowitz JS. Tracing "Fearbola": psychological predictors of anxious responding to the threat of Ebola. Cognit Ther Res. 2015;39(6):816-25. https://doi.org/10.1007/s10608-0159701-9

24. Wheaton MG, Abramowitz JS, Berman NC, Fabricant LE, Olatunji BO Psychological predictors of anxiety in response to the H1N1 (swine flu) pandemic. Cogn Ther Res. 2011:36(3):210-8.

25. Ahorsu DK, Lin CY, Imani V, Saffari M, Griffiths MD, Pakpour AH. The fear of COVID-19 scale: development and initial validation. Int J Ment Heal Addict. 2020:1-9.

26. Umemneku Chikere CM, Wilson K, Graziadio S, Vale L, Allen AJ. Diagnostic test evaluation methodology: a systematic review of methods employed to evaluate diagnostic tests in the absence of gold standard an update. PLoS One. 2019;14(10):e0223832. https://doi.org/10.1371/journa pone.0223832.

27. Palayew A, Norgaard O, Safreed-Harmon K, Andersen TH, Rasmussen LN, Lazarus JV. Pandemic publishing poses a new COVID-19 challenge. Nat Hum Behav. 2020;4(7):666-9. https://doi.org/10.1038/s41562-020-0911-0.

28. Raynaud M, Zhang H, Louis K, Goutaudier V, Wang J, Dubourg Q, et al. COVID-19-related medical research: a meta-research and critical appraisal. BMC Med Res Methodol. 2021;21(1):1-11.

29. Boutron I, Chaimani A, Meerpohl JJ, Hróbjartsson A, Devane D, Rada G, et al. The COVID-NMA project: building an evidence ecosystem for the COVID-19 pandemic. Ann Intern Med. 2020;173(12):1015-7. https://doi.org/10.7326/M2 $0-5261$

30. Rada G, Pérez D, Araya-Quintanilla F, Ávila C, Bravo-Soto G, Bravo-Jeria R, et al. Epistemonikos: a comprehensive database of systematic reviews for health decision-making. BMC Med Res Methodol. 2020:20(1):286. https://doi. org/10.1186/s12874-020-01157-x

31. Lorenc T KC, Raine G, Shemilt I, Sutcliffe K, D'Souza P, Burchett $H_{1}$, Hinds K MW, Melton H, Richardson M, South E, Stansfield C TS, Kwan I, Wright K, 
Sowden A, Thomas J COVID-19: living map of the evidence. . London: EPPICentre, Social Science Research Unit USRI, University College London; 2020.

32. Vist $G$, Muller A, Flottorp S, Himmels J, Van de Velde S, Smedslund G, et al. A systematic and living evidence map on COVID-19. Oslo: Norwegian Institute of Public Health; 2020.

33. Whiting PF, Rutjes AW, Westwood ME, Mallett S, Deeks JJ, Reitsma JB, et al. QUADAS-2: a revised tool for the quality assessment of diagnostic accuracy studies. Ann Intern Med. 2011;155(8):529-36. https://doi.org/10.7326/0003-4 819-155-8-201110180-00009.

34. Arpaci I, Karatas K, Baloglu M. The development and initial tests for the psychometric properties of the COVID-19 phobia scale (C19P-S). Pers Individ Dif. 2020;164:110108. https://doi.org/10.1016/j.paid.2020.110108.

35. Nguyen HT, Do BN, Pham KM, Kim GB, Dam HTB, Nguyen TT, et al. Fear of COVID-19 Scale-Associations of Its Scores with Health Literacy and HealthRelated Behaviors among Medical Students. Int J Environ Res Public Health. 2020;17(11)

36. Zolotov Y, Reznik A, Bender S, Isralowitz R. COVID-19 fear, mental health, and substance use among Israeli University students. Int J Ment Heal Addict. 2020. https://doi.org/10.1007/s11469-020-00351-8.

37. StataCorp. StatStata Statistical Software: Release 16. College Station: StataCorp LLC; 2019

38. Alyami M, Henning M, Krageloh CU, Alyami H. Psychometric evaluation of the Arabic version of the fear of COVID-19 scale. Int J Ment Heal Addict. 2020:1-14.

39. Arpaci I, Karataş K, Baloğlu M. The development and initial tests for the psychometric properties of the COVID-19 Phobia Scale (C19P-S). Pers Individ Differ. 2020;164:110108.

40. Bitan DT, Grossman-Giron A, Bloch Y, Mayer Y, Shiffman N, Mendlovic S. Fear of COVID-19 scale: psychometric characteristics, reliability and validity in the Israeli population. Psychiatry Res. 2020;113100:1-5.

41. Chang KC, Hou WL, Pakpour AH, Lin CY, Griffiths MD. Psychometric testing of three COVID-19-related scales among people with mental illness. Int J Ment Heal Addict. 2020. https://doi.org/10.1007/s11469-020-00361-6.

42. Feng LS, Dong ZJ, Yan RY, Wu XQ, Zhang L, Ma J, et al. Psychological distress in the shadow of the COVID-19 pandemic: preliminary development of an assessment scale. Psychiatry Res. 2020;291:113202. https://doi.org/10.1 016/j.psychres.2020.113202.

43. Haktanir A, Seki T, Dilmac B. Adaptation and evaluation of Turkish version of the fear of COVID-19 scale. Death Studies. 2020:1-9. https://doi.org/10.1080/ 07481187.2020 .1773026$.

44. Huarcaya-Victoria J, Villarreal-Zegarra D, Podestà A, Luna-Cuadros MA. Psychometric properties of a Spanish version of the fear of COVID-19 scale in general population of Lima, Peru. Int J Ment Heal Addict. 2020:1-14.

45. Mejia CR, Ticona D, Rodriguez-Alarcon JF, Campos-Urbina AM, Catay-Medina $J B$, Porta-Quinto T, et al. The media and their informative role in the face of the coronavirus disease 2019 (COVID-19): Validation of fear perception and magnitude of the issue (MED-COVID-19). Elect J Gen Med. 2020;17(6):1-6.

46. Pang NTP, Kamu A, Hambali NLB, Mun HC, Kassim MA, Mohamed NH, et al. Malay version of the fear of COVID-19 scale: validity and reliability. Int J Ment Heal Addict. 2020:1-10

47. Perz CA, Lang BA, Harrington R. Validation of the fear of COVID-19 scale in a US College sample. Int J Ment Heal Addict. 2020. https://doi.org/10.1007/ s11469-020-00356-3.

48. Reznik A, Gritsenko V, Konstantinov V, Khamenka N, Isralowitz R. COVID-19 fear in Eastern Europe: validation of the fear of COVID-19 scale. Int J Ment Heal Addict. 2020:1-6.

49. Sakib N, Bhuiyan AKMI, Hossain S, Al M, Firoj, Hosen I, et al. Psychometric Validation of the Bangla Fear of COVID-19 Scale: Confirmatory Factor Analysis and Rasch Analysis. Int J Ment Heal Addict. 2020;(11):1-12.

50. Satici B, Gocet-Tekin E, Deniz ME, Satici SA. Adaptation of the fear of COVID19 scale: its association with psychological distress and life satisfaction in Turkey. Int J Ment Heal Addict. 2020:1-9.

51. Soraci P, Ferrari A, Abbiati Francesco A, Del F, de Elena P, et al. Validation and psychometric evaluation of the Italian version of the fear of COVID-19 scale. Int J Ment Heal Addict. 2020:1-10.

52. Tsipropoulou V, Nikopoulou VA, Holeva V, Nasika Z, Diakogiannis I, Sakka S, et al. Psychometric properties of the Greek version of FCV-19S. Int J Ment Heal Addict. 2020. https://doi.org/10.1007/s11469-020-00319-8.

53. Swets JA. ROC analysis applied to the evaluation of medical imaging techniques. Investig Radiol. 1979;14(2):109-21. https://doi.org/10.1097/ 00004424-197903000-00002.
54. Schimmenti A, Starcevic V, Giardina A, Khazaal Y, Billieux J. Multidimensional assessment of COVID-19-related fears (MAC-RF): a theory-based instrument for the assessment of clinically relevant fears during pandemics. Front Psychiatry. 2020;11:748. https://doi.org/10.3389/fpsyt.2020.00748.

55. Mertens G, Duijndam S, Smeets T, Lodder P. The latent and item structure of COVID-19 fear: A comparison of four COVID-19 fear questionnaires using SEM and network analyses. PsyArXiv. 2020:1-42. preprint.

56. Van Bortel T, Basnayake A, Wurie F, Jambai M, Koroma AS, Muana AT, et al. Psychosocial effects of an Ebola outbreak at individual, community and international levels. Bull World Health Organ. 2016;94(3):210-4. https://doi. org/10.2471/BLT.15.158543.

57. Gover AR, Harper SB, Langton L. Anti-Asian hate crime during the COVID-19 pandemic: exploring the reproduction of inequality. Am J Crim Justice. 2020:1-21.

58. Rzymski P, Nowicki M. COVID-19-related prejudice toward Asian medical students: a consequence of SARS-CoV-2 fears in Poland. J Infect Public Health. 2020;13(6):873-6. https://doi.org/10.1016/j.jph.2020.04.013.

59. Gray C, Hansen K. Did Covid-19 lead to an increase in hate crimes towards Chinese people in London? London: UCL Social Research Institute, Science QS; 2020. Contract No.: 20-05

60. Octavius GS, Silviani FR, Lesmandjaja A, Juliansen A. Impact of COVID-19 on adolescents' mental health: a systematic review. Middle East Curr Psychiat. 2020;27(1):1-8.

61. De Kock JH, Latham HA, Leslie SJ, Grindle M, Munoz SA, Ellis L, et al. A rapid review of the impact of COVID-19 on the mental health of healthcare workers: implications for supporting psychological well-being. BMC Public Health. 2021;21 (1):104. https://doi.org/10.1186/s12889-020-10070-3.

62. Deeks JJ, Dinnes J, Takwoingi Y, Davenport C, Spijker R, Taylor-Phillips S, et al. Antibody tests for identification of current and past infection with SARS-CoV-2. Cochrane Database Syst Rev. 2020;6.

63. Arevalo-Rodriguez I, Steingart KR, Tricco AC, Nussbaumer-Streit B, Kaunelis D, Alonso-Coello P, et al. Current methods for development of rapid reviews about diagnostic tests: an international survey. BMC Med Res Methodol. 2020;20(1):115. https://doi.org/10.1186/s12874-020-01004-z.

\section{Publisher's Note}

Springer Nature remains neutral with regard to jurisdictional claims in published maps and institutional affiliations.
Ready to submit your research? Choose BMC and benefit from:

- fast, convenient online submission

- thorough peer review by experienced researchers in your field

- rapid publication on acceptance

- support for research data, including large and complex data types

- gold Open Access which fosters wider collaboration and increased citations

- maximum visibility for your research: over $100 \mathrm{M}$ website views per year

At BMC, research is always in progress.

Learn more biomedcentral.com/submissions 